\title{
Identification of the 187 bp EphA7 Genomic DNA as the Dorsal Midline-Specific Enhancer of the Diencephalon and Mesencephalon
}

\author{
Yujin Kim, Eunjeong Park, and Soochul Park*
}

\begin{abstract}
EphA7 is a key molecule in regulating the development of the dien- and mesencephalon. To get insight into the mechanism of how EphA7 gene expression is regulated during the dorsal specification of the dien- and mesencephaIon, we investigated the cis-acting regulatory sequence driving EphA7 to the dorsal midline of the dien- and mesencephalon. Transgenic LacZ reporter analysis, using overlapping EphA7 BACs, was used to narrow down the dorsal midline-specific enhancer, revealing the $25.3 \mathrm{~kb}$ genomic region as the enhancer candidate. Strikingly, this genomic DNA was located far downstream of the EphA7 transcription start site, $+302.6 \mathrm{~kb}$ to $+327.9 \mathrm{~kb}$. Further enhancer mapping, using comparative genomic analysis and transgenic methods, showed that the 187 bp genomic DNA alone, approximately $305 \mathrm{~kb}$ downstream of the EphA7 transcription start site, was sufficient to act as the dorsal midline-specific enhancer of EphA7. Importantly, our results indicate that the 187 bp dorsal midline-specific enhancer is critically regulated by homeobox transcription factors during the development of the dien- and mesencephalon.
\end{abstract}

\section{INTRODUCTION}

Expression studies using Eph-Fc or ephrin-Fc fusion proteins reveal that the developing embryo is subdivided into domains demarcated by reciprocal and mutually exclusive expressions of Eph receptors and its corresponding ligands and ephrins (Gale et al., 1996; Park, 2013; Park et al., 2013). For example, EphA receptors are predominantly expressed in the dorsal region of the diencephalon and anterior mesencephalon, whereas ephrin-A ligands express in the posterior mesencephalon. This reciprocal compartmentalization strongly suggests that

Department of Biological Science, Sookmyung Women's University, Seoul 140-742, Korea

*Correspondence: scpark@sookmyung.ac.kr

Received 19 August, 2015; accepted 8 September, 2015; published online 4 November, 2015

Keywords: dorsal midline, enhancer, EphA7
Eph receptors and ephrins may be involved in the formation of spatial boundaries which facilitate the precise and dynamic organization of the developing brain. In this respect, the identification of regulatory sequences driving Eph or ephrin gene expression to a specific region would be important for understanding how morphogenes or transcription factors, could subdivide a brain region through expression regulation of different target genes during early brain development.

Evidence suggests that EphA7 plays a pivotal role in the development of the central nervous system. For example, it was reported that microdeletion of 6q16.1, encompassing EphA7 in a child, correlates with neurodevelopmental abnormalities (Traylor et al., 2009). In addition, it was shown that EphA7 mRNA is co-expressed with its cognate ligand ephrin-A5 at the edges of the dorsal neural folds, and that EphA7 mediates adhesive interaction with ephrin-A5 during the closure of neural tubes (Holmberg et al., 2000). More importantly, EphA7 null mutant embryos displayed an increase in cortical size and exencephalic overgrowth of the forebrain, suggesting it plays a role in inducing apoptotic cell death in neuroepithelial cells (Depaepe et al., 2005). Interestingly, the EphA7 expression pattern in the dorsal midline of the dien- and mesencephalon is somewhat consistent with that of Wnt1, which is essential for dorsoventral patterning of the neural tube. Data indicate that the dorsal midline of the vertebrate neural tube is required for the specification of dorsal cell fates, in the central nervous system (Lee and Jessell, 1999). For example, Msx genes encoding homeodomain transcription factors are expressed dorsally in the neural tube of all species (Houzelstein et al., 1997). At the neural fold stage, Msx1 is expressed in the future dorsal neural cells at the ectoderm/neuroectoderm boundary. Thereafter, at the neural tube closure stage, Msx1 expression is restricted in the CNS to the dorsal midline along its entire length. In addition, Msx genes appear to be intermediate between BMP and Wnt, and they are required to induce the expression of Wnt1 in the dorsal midline, and maintain the integrity of these cells (Bach et al., 2003). These findings suggest that EphA7 gene expression may be regulated by the BMP-Msx-Wnt genetic pathway during the dorsal specification of the dien- and mesencephalon.

To date, little is known about the upstream regulators driving the EphA7 gene expression to the dorsal midline of the dienand mesencephalon. Identification of the cis-acting regulatory sequences for EphA7 is important for delineating the genetic 

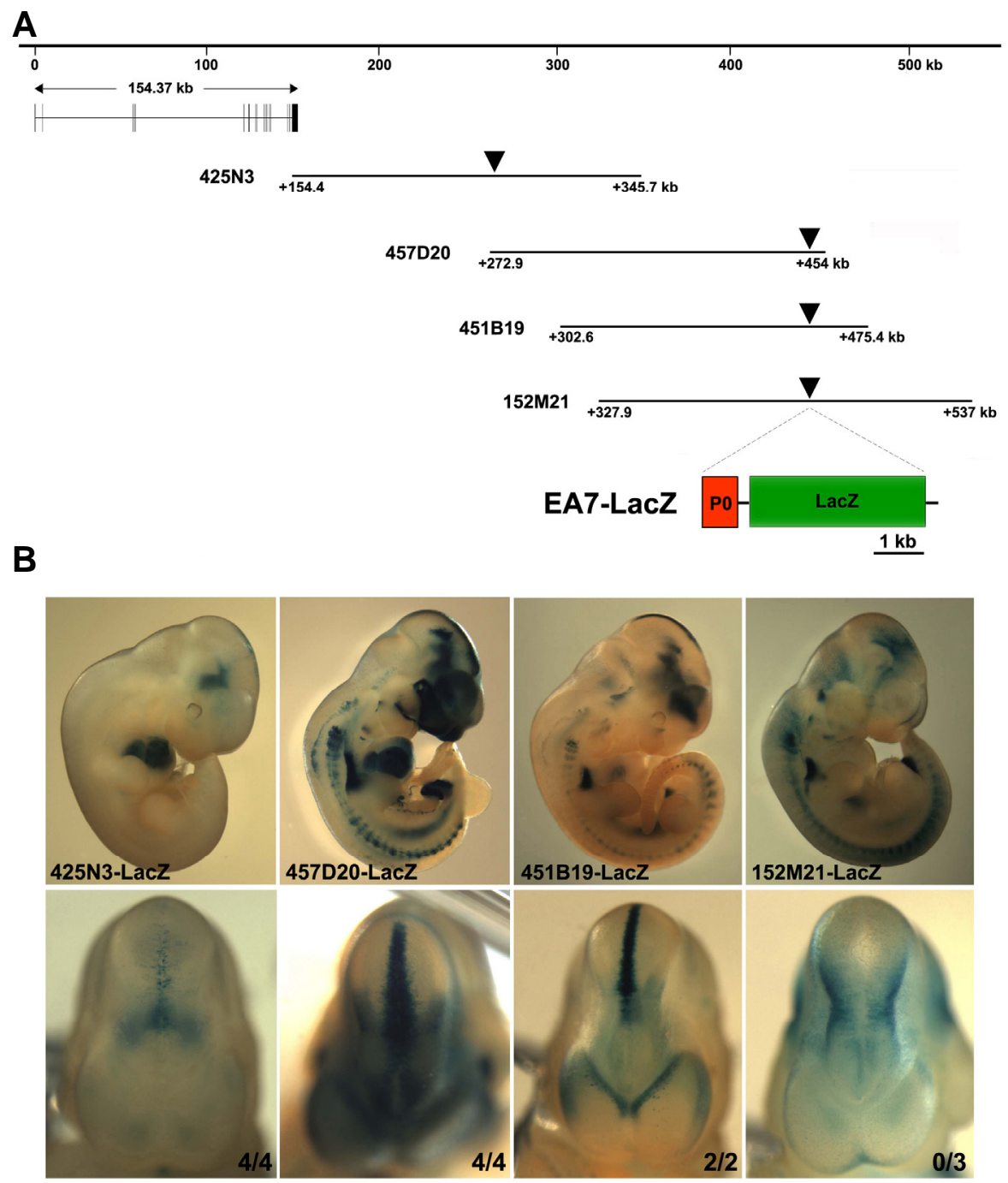

Fig. 1. Mapping of the dorsal midline-specific EphA7 enhancer using overlapping BACs. (A) Genomic map showing the distribution of overlapping BAC clones spanning $0.5 \mathrm{Mb}$ downstream of EphA7. Endogenous EphA7 promoter (P0) and LacZ reporter genes were inserted into each BAC using bacterial homologous recombination methods (the insertion site was marked by arrowhead). (B) BAC transgenic embryos harvested at E10.5 were collected for whole mount X-gal staining. A representative embryo in each group was selected for lateral (upper panels) or top views (lower panels). The number of embryos exhibiting the dorsal midline-specific reporter activity over the total number of transgenic embryos was indicated in each panel. For the BAC transgenic embryos carrying $425 \mathrm{~N} 3$, the dorsal midline-specific activity is $100 \%$ (4/4), although LacZ expression was weakly detected (first panels). pathway for the dorsoventral patterning of the dien- and mesencephalon. In this study, we used the BAC transgenic approach, and comparative genomic analysis, to identify the 187 bp genomic DNA as a final candidate for the dorsal midlinespecific enhancer of EphA7.

\section{MATERIALS AND METHODS}

Construction of targeting vectors for bacterial homologous recombination

Homologous arms A and B were subcloned into a reporter vector containing the EphA7 endogenous promoter, a LacZ reporter gene and SV40 poly(A) signal. Homologous arms for each EphA7 BAC, 425N3, 457D20, 451B19 and 152M21, were synthesized by PCR using the following primer sets: 425N3 (A arm: 5'-GTTTTAGTTCCCTGCGGACC-3' and 5'-CAAAAGCAACTT GGGGAGGAA-3', B arm: 5'-ACTTTTGATGGGAGGATGCC-3' and 5'-GAACAAGAGGGCCTGCTCAT-3'), 457D20, 451B19 and 152M21 (A arm: 5'-TTTTGTGTCAACTTGAC-3' and 5'CTCGAGCCATCAATTGC-3', B arm: 5'-ACTGAAGGGGTCA TGCAGAA-3' and 5'-AATTTTATTGAATATACT-3'). Targeting vectors were constructed on the backbone of the pGEM11Z vector (Promega) as follows: First, pGEM11Z was digested with $\mathrm{Xbal}$ and then a FRT-Kana-FRT cassette was inserted into this site. Second, the EphA7 endogenous promoter and LacZ containing the SV40 polyadenylation site were cloned into the Sall site in front of a FRT-Kana-FRT cassette in pGEM11Z. Then, homologous $A$ and $B$ arms were cloned into the Sfil/Spel and NotI/Nsil site in pGEM11Z, respectively. These targeting vectors were digested with $\mathrm{Nsil} / \mathrm{Sfil}$ and then the insert was isolated through separation on a $0.8 \%$ low-melting agarose gel.

All candidate DNA for the EphA7 enhancer were amplified by PCR using the following primer sets: ECR (5'-CTATAGTTTTTAA GTGGAAA-3' and 5'-CAAGGACCCTCCCAGGATTG-3'); ECR1 (5'-CTATAGTTTTTAAGTGGAAA-3' and 5'-AAGAAAGCGGGT AAGAGATT-3'); ECR2 (5'-TTGTCTTCTAGTATTTTCAA-3' and 5'-ATACAGTTACTTCTCTCGTG-3'); ECR3 (5'-TTTTTGGCTCA ACAAAGCTTG-3' and 5'-AAGGACCCTCCCAGGATTG-3').

Bacterial homologous $\mathrm{BAC}$ recombination was performed as described previously (Kim et al., 2007).

Generation BAC transgenic mice and plasmid transgenic embryos

Modified BAC DNA was prepared using the large-construct kit (Qiagen). The BAC DNA for microinjection was confirmed on a gel and diluted with injection buffer as described previously (Kim et al., 2007). The BAC DNA was then injected into 200 pronuclei of fertilized oocytes of C57BL/6 mice, as described 
A

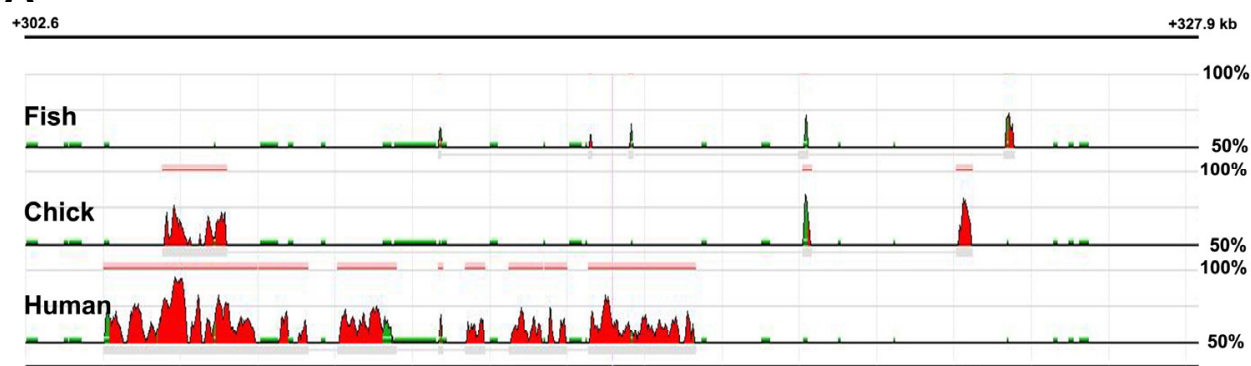

B

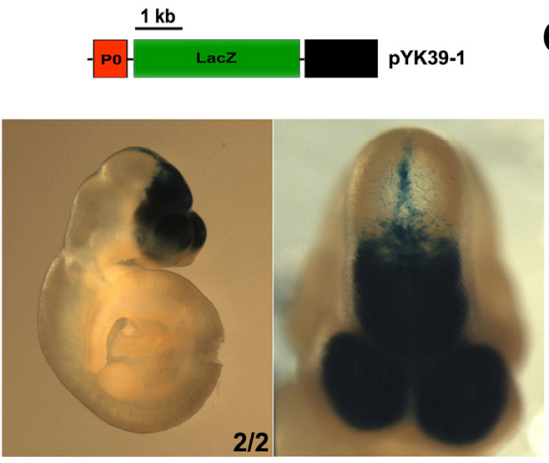

C

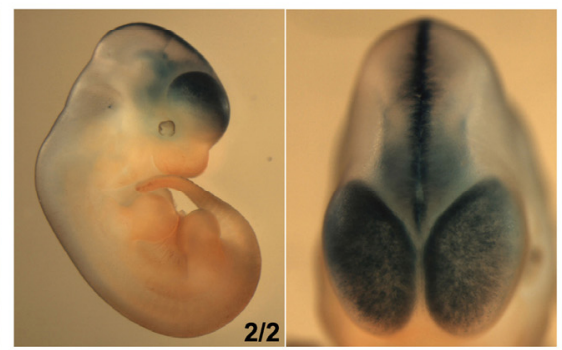

Fig. 2. Identification of the 1.4 $\mathrm{kb}$ dorsal midline-specific EphA7 enhancer. (A) ECR analysis comparing the alignment of mouse genomic sequences between $+302.6 \mathrm{~kb}$ and $+327.9 \mathrm{~kb}$ downstream of the EphA7 transcription start site in human, chicken, and fish. A $1.4 \mathrm{~kb}$ ECR region (> $40 \%$ similarity over a minimum of $100 \mathrm{bp}$ ) among two ECR regions conserved in chicks was located $+305.6 \mathrm{~kb} \sim 307 \mathrm{~kb}$ downstream of the EphA7 transcription start site. (B) A 1.4 $\mathrm{kb}$ ECR region was amplified by PCR and cloned into the front or the back of the transgenic vector carrying EphA7 endogenous promoter $(\mathrm{PO})$ and the LacZ reporter gene. Mouse embryos were harvested at E10.5 for whole mount X-gal staining. Note that LacZ expression was detected in the dorsal midline of the dien- and mesencephalon regardless of the position of the $1.4 \mathrm{~kb} E C R$ DNA in the vector. previously (Gong et al., 2002; Kim et al., 2007). BAC transgenic embryos were identified by PCR, using the primers 5'ACTTCGGAGCAAACAGCATCTA-3' and 5'-CGGAAACCAGG CAAAGCGCCAT-3'. Plasmid transgenic embryos were identified by PCR using the primers 5'- GTTACAATAAGGCAATA GCATCACA-3' and 5'- AACTACCAATCACCGACCCC-3'.

\section{Histology and $X$-gal staining}

$X$-gal embryos to be stained were dissected in phosphate buffered saline (PBS), fixed in $0.2 \%$ glutaraldehyde, and subjected to the washing and staining procedure as described previously (Koo et al., 2003; Shim et al., 2007).

\section{RESULTS}

Isolation of the $25.3 \mathrm{~kb}$ genomic DNA as the dorsal midline-specific EphA7 enhancer

Our previous study using an enhancer trap assay suggested that 457D20 EphA7 BAC contained the putative enhancer directing EphA7 gene expression to the dorsal midline of the dien- and mesencephalon (Kim and Park, 2011). To further locate the dorsal midline-specific enhancer of EphA7, we used the bacterial homologous recombination method to target LacZ reporter to two overlapping BACs: 425N3 and 457D20 (Fig. 1A). Each targeting vector contained $0.8 \mathrm{~kb}$ of genomic DNA acting as a basal promoter (P0), which is positioned upstream of the EphA7 transcription initiation site. This basal promoter is followed by a LacZ reporter and a kanamycin selection marker as described before (Kim and Park, 2011). Either side of each targeting vector contains distinct EphA7 genomic sequences, which allow specific recombination between the targeting vector and BAC. The genomic structure of each recombinant BAC was examined through Southern blot analysis and then as- sayed for its reporter activity in transgenic mouse embryos at embryonic day (E) 10.5. As expected, 457D20-LacZ BAC transgenic embryos revealed a specific expression pattern of EphA7 in the dorsal midline of the dien- and mesencephalon (100\% enhancer activity) (Fig. 1B, second panels). Importantly, 425N3-LacZ BAC transgenic embryos also revealed weak but specific expression of LacZ along the dorsal midline of the dienand mesencephalon (100\% enhancer activity). These results strongly suggest that the genomic region spanning from +272.9 $\mathrm{kb}$ to $+345.7 \mathrm{~kb}$ is likely to contain the EphA7 enhancer specific for the dorsal midline.

Identification of the $187 \mathrm{bp}$ genomic DNA as the dorsal midline-specific EphA7 enhancer

To further isolate the dorsal midline-specific enhancer within this genomic region, we generated two additional recombinant BACs, 451B19-LacZ and 152M21-LacZ, using the same vector used for generating 457D20-LacZ recombinant BAC (Fig. 1A). Analysis of transgenic embryos carrying these recombinant BACs demonstrated that 451B19 BAC, but not 152M21 BAC, contained the dorsal midline-specific enhancer (Fig. 1B, third and fourth panels), raising the possibility that the enhancer is located in $25.3 \mathrm{~kb}$ genomic region between $+302.6 \mathrm{~kb}$ and $+327.9 \mathrm{~kb}$ downstream from the EphA7 transcription start site.

To further identify the dorsal midline-specific EphA7 enhancer we used comparative genomic analysis of different organisms, and found an evolutionary conserve region (ECR) (> 40\% identity over $100 \mathrm{bp}$ ) which was highly conserved between mice and chicks, distantly related organisms, within the $25.3 \mathrm{~kb}$ mouse genomic region (Fig. 2A). To determine whether this ECR DNA (from $+305.6 \mathrm{~kb}$ to $+307 \mathrm{~kb}$ ) acts as the dorsalmidline-specific enhancer, the corresponding mouse genomic DNA was amplified by PCR and subcloned into the expression 
$\boldsymbol{A}$

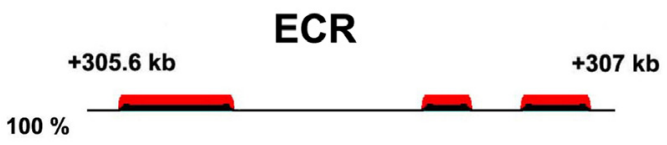

B

$50 \%$
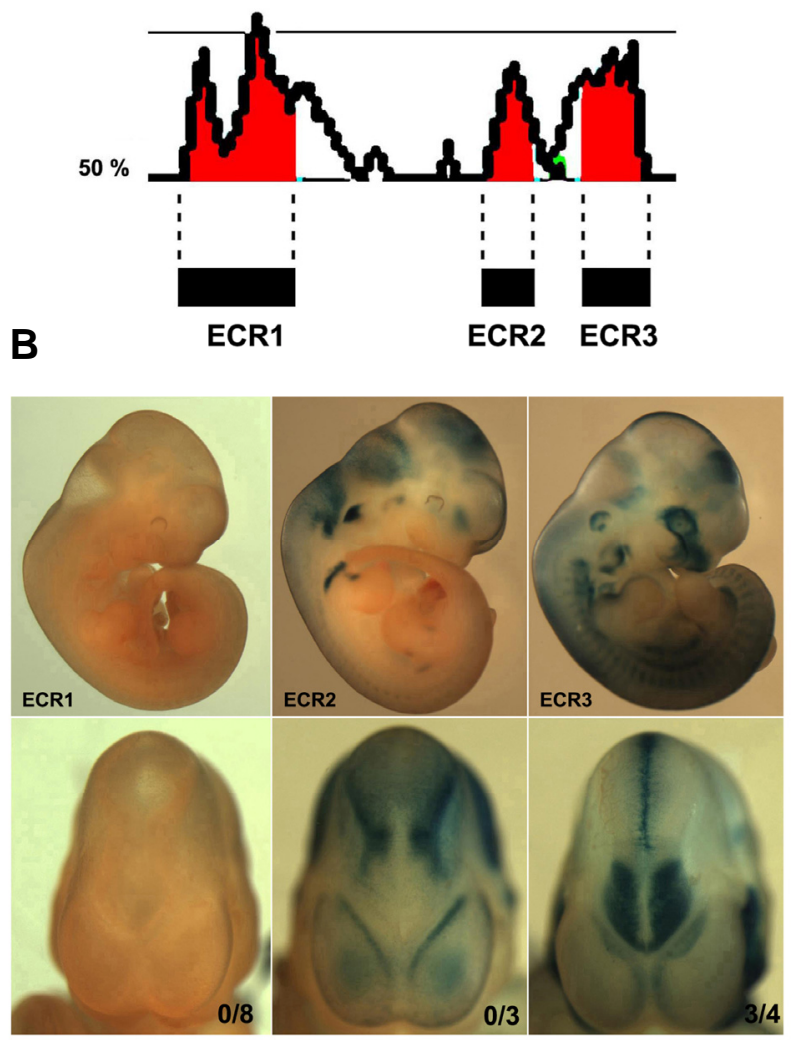

Fig. 3. Identification of the $187 \mathrm{bp}$ dorsal midline-specific EphA7 enhancer. (A) Schematic representation for three highly conserved regions (ECR1 3; $>70 \%$ similarity over a minimum of $100 \mathrm{bp}$ ) within the $1.4 \mathrm{~kb}$ ECR. (B) Each of the three conserved ECR regions was amplified by $\mathrm{PCR}$ and subcloned into the transgenic vector carrying EphA7 endogenous promoter and LacZ reporter gene. Note that transgenic embryos carrying ECR3 showed LacZ expression in the dorsal midline of diencephalon and mesencephaIon.

cassette containing an EphA7 basal promoter and a LacZ reporter gene (Fig. 2B, top). As expected, the analysis of transgenic embryos carrying the enhancer construct revealed that $1.4 \mathrm{~kb}$ genomic DNA contained the dorsal midline-specific enhancer. Its activity was not dependent on the enhancer position in the expression construct (Fig. 2B, bottom panels). Comparative genomic analysis of this $1.4 \mathrm{~kb}$ enhancer DNA also revealed that ECR can be further divided into three regions, termed ECR1, ECR2 and ECR3. Each DNA sequence may be important for the dorsal midline enhancer function (Fig. 3A). Therefore, each of these ECR regions was amplified by PCR and subcloned into the expression vector containing a EphA7 basal promoter and a LacZ reporter. Importantly, we found that transgenic embryos carrying ECR3, but not ECR1 or 2, displayed LacZ expression in the dorsal midline of the dien- and mesencephalon ( $75 \%$ enhancer activity) (Fig. 3B). These results strongly indicate that the $187 \mathrm{bp}$ genomic DNA located in between $+306.8 \mathrm{~kb}$ and $+307 \mathrm{~kb}$ downstream of the EphA7 transcription start site is likely to represent the dorsal midlinespecific enhancer of EphA7.

The binding sites for homeobox transcription factors are critical for the EphA7 enhancer activity

We further isolated a chick's genomic DNA corresponding to mouse ECR3 and investigated whether it can act as the dorsal midline-specific enhancer in a transgenic mouse embryo (Figs. $3 \mathrm{C}$ and $4 \mathrm{~A}$ ). Importantly, our results strongly suggest that chick ECR3 was also able to direct LacZ expression to the dorsal midline of the dien- and mesencephalon although its reporter activity $(\sim 17 \%)$, which was much lower than mouse ECR3 $(\sim 75 \%)$ (Fig. 4B; Fig. 4C, first panel). Closer examination of mouse ECR3 and chick ECR3 revealed that a consensus sequence (TAAT) for homeobox transcription factors is frequently found but also highly conserved (Fig. 4A). To investigate whether the conserved transcription factor binding sites are functionally important for the 187 bp ECR3 enhancer activity, three putative transcription factor binding sites were selected for deletion. The deleted ECR3 DNA was examined for its effect on the enhancer activity by creating transgenic mouse embryos (Figs. 4A and 4B). Whereas site 1 (a putative binding site for TCF1) was still effective in inducing LacZ expression in the dorsal midline (21\%) (Fig. 4C, second panel), deletion of site 2 displayed only marginal and weak enhancer activity, restricted to the dorsal midline of the mesencephalon ( 20\%) (Fig. 4C, third panel). More importantly, the deletion of site 3 resulted in no enhancer activity in transgenic embryos (Fig. 4C, fourth panel). Together, these results suggest that homeobox transcription factors are likely important for binding to the dorsal midline-specific enhancer of the dien- and mesencephalon.

\section{DISCUSSION}

In this study, we used the bacterial homologous recombination method to generate various recombinant BACs and transgenic mouse embryos to map the EphA7 enhancer driving the LacZ reporter to the dorsal midline of the dien- and mesencephalon. In addition, the comparative genomic analysis and ECR-based transgenic reporter analysis led to the identification of 187 bp genomic DNA as a final candidate for the dorsal midline-specific enhancer of EphA7. Strikingly, this dorsal midline enhancer existed in between $+306.8 \mathrm{~kb}$ and +307 kb, far downstream from the EphA7 transcription start site. Expression patterns of EphA7 in the dorsal midline of the dien- and mesencephalon is similar to that of BMP1 and Wnt1, which play critical roles in the dorsoventral patterning of the neural tube (Furuta et al., 1997; McMahon et al., 1992). We became interested in analyzing the role of two consensus binding sites in the ECR3 DNA: Lef1/TCF and Msx1. The Lef1, high mobility group (HMG) family transcription factor, interacts with beta-catenin and is a nuclear effector of Wnt1 (Behrens et al., 1996; Molenaar et al., 1996; van de Wetering et al., 1997). In addition, Lef1 is specifically expressed in the dorsal midline of the dien- and mesencephalon (Mailleux et al., 2002; Shimogori et al., 2010). However, our deletion analysis of the transcription factor binding sites revealed that the ECR3 DNA, lacking the putative Lef1 consensus binding site, was still capable of driving LacZ reporter to the dorsal midline, suggesting that ECR3 is not a direct target for Wnt1 signaling. The initial expression of Msx1, a homeodoman transcription factor in the neural folds, is known to be the consequence of 
A

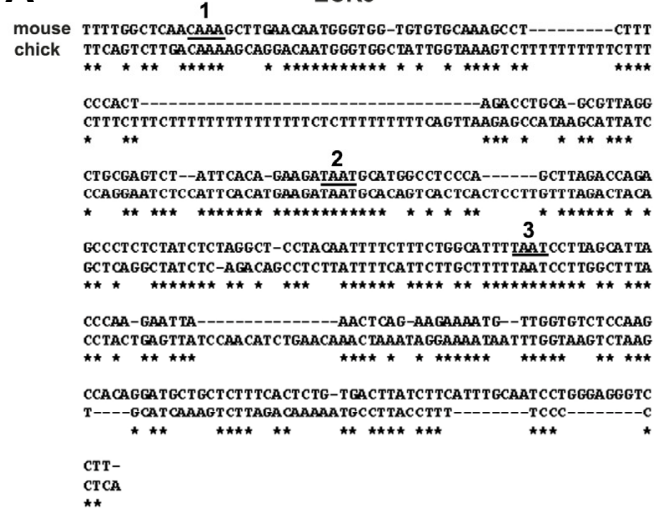

B

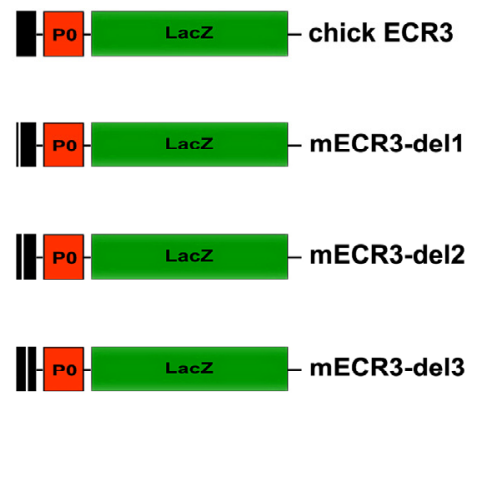

C

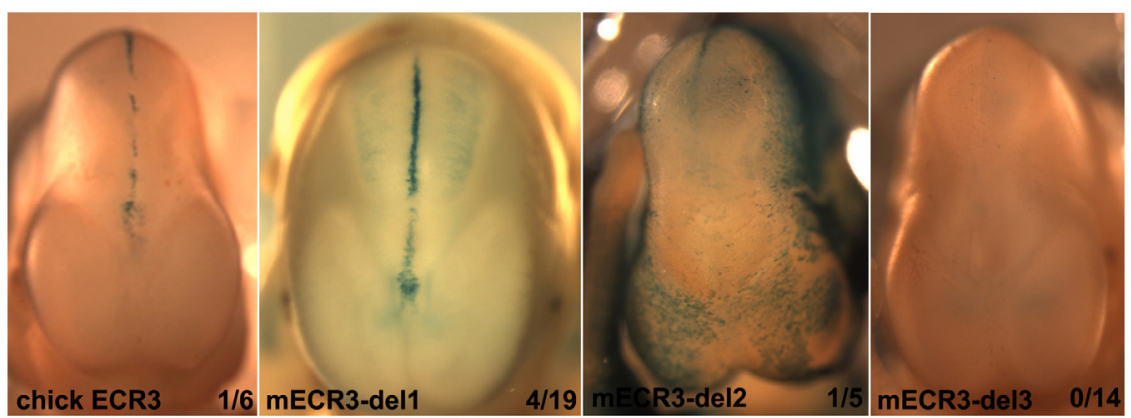

Fig. 4. The binding sites for homeodomain transcription factors are critical for the $187 \mathrm{bp}$ dorsal midline-specific enhancer activity. (A) The ECR3 sequence of mice was compared with that of chicks to reveal highly conserved transcription factor binding sites. Site 1 , a consensus binding site for Lef1/TCF, HMG family transcription factor. Site 2 and 3, a consensus binding site for homeodomain transcription factors. (B) The chick ECR3 was amplified by PCR and subcloned into the transgenic vector carrying EphA7 endogenous promoter and LacZ reporter gene (top). The mouse ECR3 lacking each of consensus binding sites was also subcloned into the transgenic vector. (C) Mouse embryos were harvested at E10.5 for whole mount $X$-gal staining. Note that LacZ expression was never detected in the embryos carrying ECR3-del3, although we analyzed the enhancer activity of up to 14 transgenic embryos (fourth panel).
BMP signaling from the lateral ectoderm (Bach et al., 2003; Ramos and Robert, 2005). After closure of the neural tube, Msx genes are specifically expressed along the dorsal midline and induce the expression of Wnt1 in the dorsal midline and maintain the integrity of the dorsal midline cells. More importantly, our deletion analysis of ECR3 DNA revealed that two different binding sites for the homeobox transcription factor were critical for the dorsal midline-specific enhancer activity. In particular, deletion of site 3 (TTTTAATCCT) was responsible for impairing the dorsal midline-specific enhancer activity in transgenic embryos. Although this result strongly suggests that Msx homeodomain transcription factors interact with these putative binding sites present in ECR3, we had technical difficulties in testing this hypothesis because antibodies against Msx 1 or 2 are not effective to test whether Msx1 is directly bound to these sites in vitro or in vivo. Nevertheless, our results support that EphA7 is a direct target for homeobox transcription factors, possibly regulated by BMP signaling.

\section{ACKNOWLEDGMENTS}

This work was supported by grants (2013M3C7A1056565, 2013R1A2A2A01005037 and 2013R1A1A2058977) from the National Research Foundation of Korea (NRF).

\section{REFERENCES}

Bach, A., Lallemand, Y., Nicola, M.A., Ramos, C., Mathis, L., Maufras, M., and Robert, B. (2003). Msx1 is required for dorsal diencephalon patterning. Development 130, 4025-4036.

Behrens, J., von Kries, J.P., Kuhl, M., Bruhn, L., Wedlich, D., Grosschedl, R., and Birchmeier, W. (1996). Functional interaction of beta-catenin with the transcription factor LEF-1. Nature 382 , 638-642.

Depaepe, V., Suarez-Gonzalez, N., Dufour, A., Passante, L., Gorski, J.A., Jones, K.R., Ledent, C., and Vanderhaeghen, P. (2005). Ephrin signalling controls brain size by regulating apoptosis of neural progenitors. Nature 435, 1244-1250.

Furuta, Y., Piston, D.W., and Hogan, B.L. (1997). Bone morphogenetic proteins (BMPs) as regulators of dorsal forebrain development. Development 124, 2203-2212.

Gale, N.W., Holland, S.J., Valenzuela, D.M., Flenniken, A., Pan, L., Ryan, T.E., Henkemeyer, M., Strebhardt, K., Hirai, H., Wilkinson, D.G., et al. (1996). Eph receptors and ligands comprise two major specificity subclasses and are reciprocally compartmentalized during embryogenesis. Neuron 17, 9-19.

Gong, S., Yang, X.W., Li, C., and Heintz, N. (2002). Highly efficient modification of bacterial artificial chromosomes (BACs) using novel shuttle vectors containing the R6Kgamma origin of replication. Genome Res. 12, 1992-1998.

Holmberg, J., Clarke, D.L., and Frisen, J. (2000). Regulation of repulsion versus adhesion by different splice forms of an Eph receptor. Nature 408, 203-206.

Houzelstein, D., Cohen, A., Buckingham, M.E., and Robert, B. (1997). Insertional mutation of the mouse Msx1 homeobox gene by an nlacZ reporter gene. Mech. Dev. 65, 123-133.

Kim, Y., and Park, S. (2011). Identification of EphA7 BAC clone containing a long-range dorsal midline-specific enhancer. BMB Rep 44, 113-117.

Kim, Y., Song, E., Choi, S., and Park, S. (2007). Engineering lacZ Reporter gene into an ephA8 bacterial artificial chromosome using a highly efficient bacterial recombination system. J. Biochem. Mol. Biol. 40, 656-661.

Koo, J., Shim, S., Gu, C., Yoo, O., and Park, S. (2003). Identification of an enhancer region in the mouse ephA8 locus directing expression to the anterior region of the dorsal mesencephalon. Dev. Dyn. 226, 596-603.

Lee, K.J., and Jessell, T.M. (1999). The specification of dorsal cell 
fates in the vertebrate central nervous system. Annu. Rev. Neurosci. 22, 261-294.

Mailleux, A.A., Spencer-Dene, B., Dillon, C., Ndiaye, D., SavonaBaron, C., Itoh, N., Kato, S., Dickson, C., Thiery, J.P., and Bellusci, S. (2002). Role of FGF10/FGFR2b signaling during mammary gland development in the mouse embryo. Development 129, 53-60.

McMahon, A.P., Joyner, A.L., Bradley, A., and McMahon, J.A (1992). The midbrain-hindbrain phenotype of Wnt-1-Mnt-1- mice results from stepwise deletion of engrailed-expressing cells by 9.5 days postcoitum. Cell 69,581-595

Molenaar, M., van de Wetering, M., Oosterwegel, M., PetersonMaduro, J., Godsave, S., Korinek, V., Roose, J., Destree, O., and Clevers, H. (1996). XTcf-3 transcription factor mediates betacatenin-induced axis formation in Xenopus embryos. Cell 86, 391-399.

Park, S. (2013). Brain-region specific apoptosis triggered by Eph/ephrin signaling. Exp. Neurobiol. 22, 143-148.

Park, E., Kim, Y., Noh, H., Lee, H., Yoo, S., and Park, S. (2013). EphA/ephrin-A signaling is critically involved in region-specific apoptosis during early brain development. Cell Death Differ. 20,
169-180.

Ramos, C., and Robert, B. (2005). msh/Msx gene family in neural development. Trends Genet 21, 624-632.

Shim, S., Kim, Y., Shin, J., Kim, J., and Park, S. (2007). Regulation of EphA8 gene expression by TALE homeobox transcription factors during development of the mesencephalon. Mol. Cell Biol. $27,1614-1630$

Shimogori, T., Lee, D.A., Miranda-Angulo, A., Yang, Y., Wang, H., Jiang, L., Yoshida, A.C., Kataoka, A., Mashiko, H., Avetisyan, M., et al. (2010). A genomic atlas of mouse hypothalamic development. Nat. Neurosci. 13, 767-775.

Traylor, R.N., Fan, Z., Hudson, B., Rosenfeld, J.A., Shaffer, L.G. Torchia, B.S., and Ballif, B.C. (2009). Microdeletion of 6q16.1 encompassing EPHA7 in a child with mild neurological abnormalities and dysmorphic features: case report. Mol. Cytogenet. 2, 17.

van de Wetering, M., Cavallo, R., Dooijes, D., van Beest, M., van Es, J., Loureiro, J., Ypma, A., Hursh, D., Jones, T., Bejsovec, A., et al. (1997). Armadillo coactivates transcription driven by the product of the Drosophila segment polarity gene dTCF. Cell 88 , 789-799 